\title{
The search for neutrino bursts from supernovae at the Baksan Underground Scintillation Telescope; 36 years of exposure
}

M.M. Kochkarov*, M.M. Boliev, I.M. Dzaparova, A.N. Kurenya, Yu.F. Novoseltsev, R.V. Novoseltseva, V.B. Petkov, P.S. Striganov, V.I. Volchenko, G.V. Volchenko, A.F. Yanin

Institute for Nuclear Research of RAS

E-mail: kchkrvdrambler.ru]

\begin{abstract}
The current status of the experiment on recording neutrino bursts is presented. As the target, we use two parts of the facility with the total mass of 240 tons. Over the period of June 30, 1980 to December 31,2016 , the actual observational time is 31.27 years. No candidate for the stellar core collapse has been detected during the observation period. An upper bound of the mean frequency of core collapse supernovae in our Galaxy is $f_{\text {col }}<0.074$ year $^{-1}$ (90\% CL).
\end{abstract}

35th International Cosmic Ray Conference - ICRC2017-

10-20 July, 2017

Bexco, Busan, Korea

\footnotetext{
* Speaker.
} 


\section{Introduction}

The detection of neutrinos from the supernova SN1987A [四, 回, 目, 团] experimentally proved the critical role of neutrinos in the explosion of massive stars, as it was suggested more than 50 years ago [1], 田, 四].

Neutrinos are especially important, because they reveal the physical conditions in the star core at the instant of collapse. The SN1987A event helped to establish some aspects of the theory, namely the total energy radiated, the neutrinos temperatures and the duration of the neutrino burst [지, Q

Large long-term neutrino detectors are the most suited ones to observe the Galaxy and search for core collapse supernovae explosions. Several neutrino detectors have been observing the Galaxy in the last decades to search for stellar collapses, namely Super-Kamiokande [एँ]], Baksan [ए],

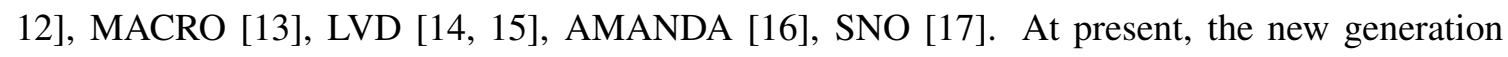
detectors, which are capable effectively to record the neutrino burst from the next $\mathrm{SN}$, are added to

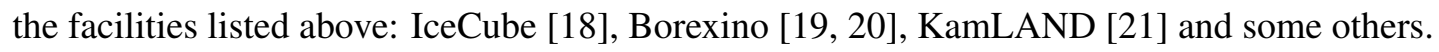

The Baksan Underground Scintillation Telescope (BUST) operates under the program of search for neutrino bursts since the mid-1980. In this paper we present the current status of the experiment and some results related to the investigation of background events and operating stability of the facility. Section 2 is the brief description of the facility. Section 3 is devoted to the method of neutrino burst detection. In Section 4 we present the joint use of two parts of the BUST with different background conditions. Conclusion is presented in Section 5.

\section{The facility}

The Baksan Underground Scintillation Telescope is located in the Northern Caucasus (Russia) in the underground laboratory at the effective depth of $8.5 \times 10^{4} \mathrm{~g} \cdot \mathrm{cm}^{-2}$ ( $850 \mathrm{~m}$ of w.e.) [22]. The facility has dimensions $17 \times 17 \times 11 \mathrm{~m}^{3}$ and consists of four horizontal scintillation planes and four vertical ones (Fig. 1). The upper horizontal plane consists of $576(24 \times 24)$ liquid scintillator

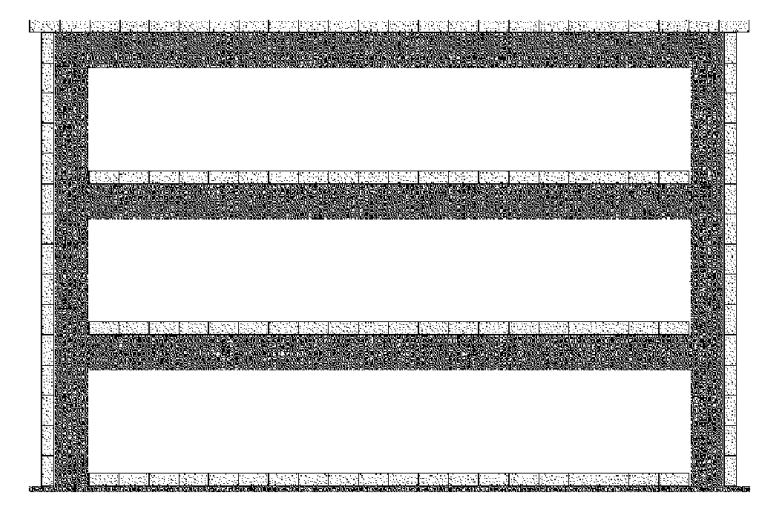

Figure 1: The Baksan underground scintillation telescope, side view.

counters of the standard type, three lower planes have $400(20 \times 20)$ counters each. The vertical planes have $15 \times 24$ and $15 \times 22$ counters. Each counter is $0.7 \times 0.7 \times 0.3 \mathrm{~m}^{3}$ in size, filled with 
an organic $C_{n} H_{2 n+2}(n \simeq 9)$ scintillator, and viewed by one photomultiplier with a photocathode diameter of $15 \mathrm{~cm}$. The distance between neighboring horizontal scintillation layers is $3.6 \mathrm{~m}$. The angular resolution of the facility is $2^{o}$, time resolution is $5 \mathrm{~ns}$.

The information from each counter is transmitted over three channels: an anode channel (which serves for amplitude measurements up to $2.5 \mathrm{GeV}$ ), a pulse channel with operation threshold $8 \mathrm{MeV}$ and $10 \mathrm{MeV}$ for the horizontal and vertical planes, respectively (the most probable energy deposition of a muon in a counter is $50 \mathrm{MeV} \equiv 1$ relativistic particle) and a logarithmic channel with a threshold $s_{o}=0.5 \mathrm{GeV}$. The signal from the fifth dynode of PM tube FEU-49 goes to a logarithmic channel where it is converted into a pulse whose length $t$ is proportional to the logarithm of the amplitude of the signal [23]].

The trigger is an operation of any counter pulse channel of the BUST.

The facility has been operating almost continuously under the program of search for neutrino bursts since the mid-1980. The total time of Galactic observation accounts for $90 \%$ of the calender time.

\section{The method of neutrino burst detection}

The BUST consists of 3184 standard autonomous counters. The total scintillator mass is 330 $\mathrm{t}$, and the mass enclosed in three lower horizontal layers (1200 standard counters) is 130 tons. The majority of the events recorded with the Baksan telescope from a supernova explosion will be produced in inverse beta decay (IBD) reactions

$$
\bar{v}_{e}+p \rightarrow n+e^{+}
$$

If the mean antineutrino energy is $E_{v_{e}}=12-15 \mathrm{MeV}$ [24, 25] the pass of $e^{+}$(produced in reaction (B.D)) will be included, as a rule, in the volume of one counter. In such case the signal from a supernova (SN) explosion will appear as a series of events from singly triggered counters (one and only one counter from 3184 operates; below we call a such event "the single event") during the neutrino burst. The search for a neutrino burst consists in recording of single events cluster within time interval of $\tau=20 \mathrm{~s}$ (according to the modern collapse models the burst duration does not exceed $20 \mathrm{~s}$ ).

The expected number of neutrino interactions detected during an interval of duration $\Delta t$ from the beginning of the collapse can be expressed as:

$$
N_{e v}^{H}=N_{H} \int_{0}^{\Delta t} d t \int_{0}^{\infty} d E F(E, t) \cdot \sigma(E) \eta(E),
$$

here $N_{H}$ is the number of free protons, $F(E, t)$ is the flux of electron antineutrinos, $\sigma(E)$ - the IBD cross section, and $\eta(E)$ - the detection efficiency. The symbol " $\mathrm{H}$ " in left side indicates that the hydrogen of scintillator is the target.

If one assumes the distance from the $\mathrm{SN}$ is $10 \mathrm{kpc}$, the total energy irradiated in neutrinos is

$$
\varepsilon_{\text {tot }}=3 \times 10^{53} \mathrm{erg}
$$

and the target mass is $130 \mathrm{t}$ (three lower horizontal layers) the expected number of single events from reaction (B.D) (we assume the total energy of the $\bar{v}_{e}$ flux is equal to $1 / 6 \times \varepsilon_{t o t}$ ) will be

$$
N_{e v}^{H} \simeq 35
$$


Flavor oscillations are unavoidable of course. However, it was recognized in recent years that the expected neutrino signal depends strongly on the oscillation scenario (see e.g. [26, 27, [28, 20]). In the absence of a quantitatively reliable prediction of the flavor-dependent fluxes and spectra it is difficult to estimate the oscillation impact on $v_{e}$ and $\bar{v}_{e}$ fluxes arriving to the Earth.

Therefore we do not discuss the effects of flavor oscillations in this paper.

Background events are radioactivity (mainly from cosmogeneous isotopes) and cosmic ray muons if only one counter from 3184 hit. The total count rate from background events is $f_{1}=$ $0.02 s^{-1}$ in internal planes (three lower horizontal layers) and $\simeq 1.5 s^{-1}$ in external ones. Therefore three lower horizontal layers are used as a target; below we call this the D1 detector (the estimation (B.4) has been made for the D1 detector).

Background events can imitate the expected signal ( $\mathrm{k}$ single events within sliding time interval $\tau)$ with a count rate

$$
p(k)=f_{1} \times \exp \left(-f_{1} \tau\right) \frac{\left(f_{1} \tau\right)^{k-1}}{(k-1) !}
$$

The treatment of experimental data (single events over a period $2001-2016 \mathrm{y} ; T_{\text {actual }}=13.67$ years) is shown by squares in Fig. $\square$ in comparison with the expected distribution according to the expression (B.5) calculated at $f_{1}=0.02 \mathrm{~s}^{-1}$. Note there is no normalization in Fig.】.

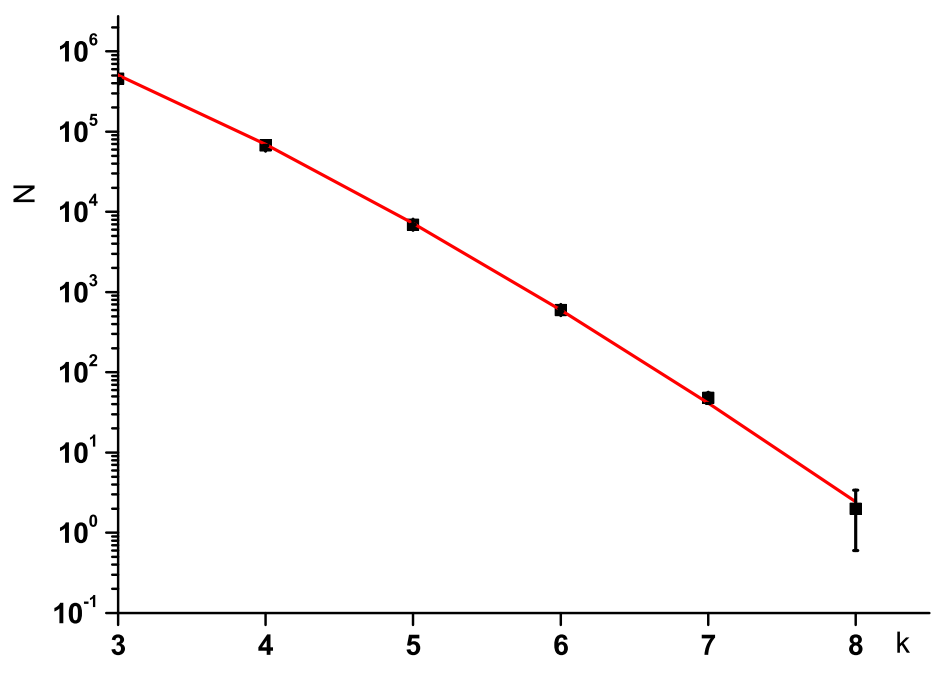

Figure 2: The number of clusters with $k$ single events within time interval of $\tau=20 \mathrm{~s}$. Squares are experimental data, the curve is the expected number according to the expression (3.5)

Background events create clusters with $\mathrm{k}=8$ with the rate $0.138 y^{-1}$. The expected number of such clusters during the time interval $\mathrm{T}=13.67 \mathrm{y}$ is 1.88 that we observe ( 2 events). The formation rate of clusters with $\mathrm{k}=9$ background events is $6.9 \times 10^{-3} y^{-1}$, therefore the cluster with multiplicity $k \geq k_{t h}=9$ should be considered as a neutrino burst detection. 


\subsection{Reactions on Carbon nuclei}

There are models which predict the mean neutrino energy from SN is $\bar{E}_{v_{e}}=30-40 \mathrm{MeV}$ [BO, [1]]. In such case the reactions on Carbon nuclei of the scintillator become effective and neutrinos can be detected in the BUST through interactions:

$$
\begin{aligned}
& v_{i}+{ }^{12} \mathrm{C} \rightarrow{ }^{12} C^{*}+v_{i}, \quad E_{t h}=15.1 \mathrm{MeV}, \\
& i=e, \mu, \tau, \\
& { }^{12} C^{*} \rightarrow{ }^{12} \mathrm{C}+\gamma, \quad E_{\gamma}=15.1 \mathrm{MeV}
\end{aligned}
$$

and

$$
\begin{aligned}
& v_{e}+{ }^{12} \mathrm{C} \rightarrow{ }^{12} \mathrm{~N}+e^{-}, \quad E_{t h}=17.34 \mathrm{MeV}, \\
& { }^{12} \mathrm{~N} \rightarrow{ }^{12} \mathrm{C}+e^{+}+v_{e}, \quad \tau\left({ }^{12} \mathrm{~N}\right)=15.9 \mathrm{~ms},
\end{aligned}
$$

$\tau$ is a lifetime of the nucleus ${ }^{12} N$.

If the mean energy $\bar{E}_{v}=30 \mathrm{MeV}$ the expected number of events for reactions (B.6) and (B.7) can be estimated (under conditions (3.3])) by formulae

$$
\begin{aligned}
& N_{e v 2}^{C}=16 \times \eta_{2}\left(E_{\gamma}=15 \mathrm{MeV}\right), \\
& N_{e v 3}^{C}=30 \times \eta_{3}\left(E_{v}=30 \mathrm{MeV}\right),
\end{aligned}
$$

The radiation length for our scintillator is $47 \mathrm{~g} / \mathrm{cm}^{2}$ therefore $\eta_{2} \approx 0.2$. In reaction (B.7) the BUST can detect both $e^{-}$with energy $\left(E_{v}-17\right) \mathrm{MeV}$ and $e^{+}$if the energy deposition from these particles is greater $8 \mathrm{MeV}$. In the latter case, the reaction (B.]) will have the distinctive signature: two signals separated with $1-50 \mathrm{~ms}$ time interval (dead time of the BUST is $\simeq 1 \mathrm{~ms}$ ). In reaction (B.]) the sum of energies $E_{e^{+}}+E_{v}$ is $17.3 \mathrm{MeV}$ therefore $\eta_{3} \approx 0.5-0.7$.

It should be noticed, if $\bar{E}_{v_{e}}=30-40 \mathrm{MeV}$ a noticeable percentage of neutrino reactions will cause triggering two adjacent counters.

\section{Two independent detectors}

As it follows from the estimation (B.4) the "sensitivity radius" of the D1 detector is $R_{s} \simeq 20$ kpc. To increase the sensitivity radius, we use those parts of external scintillator layers that have relatively low count rate of background events. The total number of counters in these parts is 1012 , the scintillator mass is 110 tons. We call this array the $\mathrm{D} 2$ detector, it has the count rate of single events $f_{2}=0.12 \mathrm{~s}^{-1}$. The count rates of single events in D1 and D2 detectors and the operating stability have been shown in Fig.[1].

The joint use of D1 and D2 detectors allows us to decrease the threshold multiplicity in D1 cluster $\left(k_{t h}=9\right)$ and, consequently, to increase $R_{s}$.

We use the following algorithm: in case of cluster detection with $k 1 \geq 6$ in the D1, we check the number of single events $k 2$ in the 10 -second time frame in the D2 detector. The start of the frame coincides with the start of the cluster in D1. Mass ratio of D2 and D1 detectors 1012/1200 $=0.843$ implies that for the mean value of neutrino events $k 1=6$ in D1, the mean number of neutrino events in D2 will be $\overline{k 2}=6 \times 0.843 * 0.8=4.05$ (factor 0.8 takes into account that the 


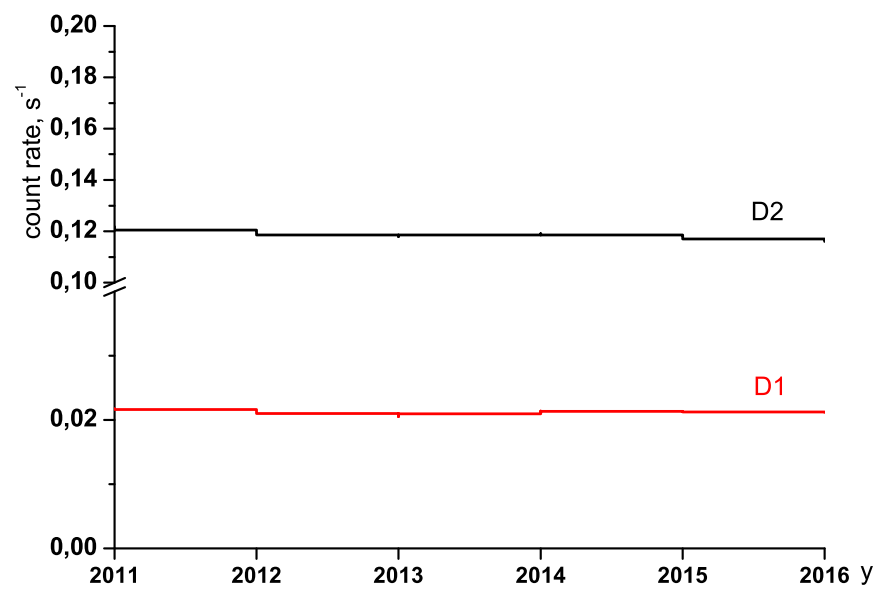

Figure 3: The count rates of single events in D1 and D2 detectors over the period 2011-2016 ys

frame duration in D2 is 10 seconds instead of 20 seconds in D1). Since the background adds $f_{2} \times 10 \mathrm{~s}=1.2$ events, we obtain finally $\overline{k 2}(\overline{k 1}=6)=4.05+1.2=5.25$.

The D1 and D2 detectors are independent, therefore the imitation probability of clusters with multiplicities k1 in D1 and k2 in D2 by background events is the product of appropriate probabilities

$$
P(k 1, k 2)=P 1(k 1) \times P 2(k 2)
$$

and we obtain $P(6,5)=0.23 y^{-1}, P(6,6)=0.045 y^{-1}$ (note $P 1$ is determined according to the expression (B.5) and $P 2$ is the Poisson distribution).

Therefore the events with $k 1 \geq 6, k 2 \geq 6$ should be considered as candidates for a neutrino burst detection (since mean values of $\mathrm{k} 1$ and $\mathrm{k} 2$ are significantly exceeded in two independent detectors simultaneously and the imitation probability of such events by background is very small). Thus we decrease the threshold value of $k 1$ from 9 to 6 and increase the sensitivity radius up to $R_{s} \simeq 24 \mathrm{kpc}$.

\section{Conclusion}

The Baksan Underground Scintillation Telescope operates under the program of search for neutrino bursts since June 30, 1980. As the target, we use two parts of the BUST (the D1 and D2 detectors) with the total mass of 240 tons. The "sensitivity radius" of the BUST (for a recording of neutrino bursts from supernovae) is $R_{s} \simeq 24 \mathrm{kpc}$.

Background events are 1) decays of cosmogeneous isotopes (which are produced in inelastic interaction of muons with the scintillator carbon and nuclei of surrounding matter) and 2) cosmic ray muons if only one counter from 3184 hit.

Over the period of June 30, 1980 to December 31, 2016, the actual observation time was 31.27 years. This is the longest observation time of our Galaxy with neutrino at the same facility. No 
candidate for the core collapse has been detected during the observation period. This leads to an upper bound of the mean frequency of gravitational collapses in the Galaxy

$$
f_{\text {col }}<0.74 y^{-1}
$$

at $90 \%$ CL. Recent estimations of the Galactic core-collapse SN rate give roughly the value $\simeq 2-5$ events per century (see e.g. [32]).

\section{References}

[1] K. Hirata, T. Kajita, M. Koshiba et al., Observation of a neutrino burst from the supernova SN1987A, Phys.Rev.Lett., 58, 1490 (1987)

[2] Bionta R.M., Blewitt G., Bratton C.B. et al. (IMB collaboration), Observation of a neutrino burst in coincidence with supernova 1987A in the large magellanic cloud, Phys.Rev.Lett., 58, 1494 (1987)

[3] E.N. Alekseev, L.N. Alekseeva, I.V. Krivosheina and V.I. Volchenko, Detection of the neutrino signal from SN, 1987A using the INR Baksan underground scintillation telescope, JETPL, 45, 589 (1987)

[4] M. Aglietta, G. Badino, G. Bologna et al., On the event observed in the Mont Blanc Underground Neutrino observatory during the occurrence of Supernova 1987A, Europhys.Lett., 3, 1315 (1987)

[5] Gamow G. and Shoenberg M., The Possible Role of Neutrinos in Stellar Evolution, Phys.Rev., 58, 1117 (1940)

[6] Zeldovich Ya.B. and Guseinov O.Kh., Neutronization of matter during collapse and the neutrino spectrum, Dokl.Akad. Nauk SSSR, 162, 791 (1965)

[7] Colgate S.A. and White R.H., The hydrodynamic behavior of supernovae explosions, Astrophys.J., 143, 626 (1966)

[8] Loredo T.J., Lamb D.Q., Bayesian Analysis of Neutrinos from Supernova SN1987A, Phys.Rev.D, 65, 063002 (2002)

[9] Pagliaroli G., Vissani F., Costantini M.L., Ianni A., Improved analysis of SN1987A antineutrino events, Astropart.Phys., 31, 163 (2009)

[10] M. Ikeda, A. Takeda, Y. Fukuda et al., (Super-Kamiokande Collaboration) "Search for Supernova neutrino bursts at Super-Kamiokande", Astrophys.J., 669, 519 (2007)

[11] E.N. Alekseev, L.N. Alekseeva, V.I. Volchenko et al., Upper bound on the collapse rate of massive stars in the Milky Way given by neutrino observations with the Baksan underground telescope, Zh. Eksp.Teor.Fiz., 104, 2897 (1993)

[12] R.V. Novoseltseva, M.M. Boliev, I.M. Dzaparova et al., The Search for Neutrino Bursts from Core Collapse Supernovae at the Baksan Underground Scintillation Telescope, in proceedings of 31th ICRC, Lodz (2009)

[13] Ambrosio M. et al. (MACRO Collaboration) Search for stellar gravitational collapses with the MACRO detector, Eur.Phys.J.C, 37, 265 (2004)

[14] Aglietta M. et al. (LVD Collaboration), The most powerful scintillator supernovae detector: LVD, Nuovo Cimento A, 105, 1793 (1992)

[15] N.Yu. Agafonova, M. Aglietta, P. Antonioli et al. (LVD collaboration), Study of the effect of neutrino oscillations on the supernova neutrino signal in the LVD detector, Astropart.Phys., 27, 254 (2007) 
[16] Ahrens J. et al. (AMANDA Collaboration), Search for supernova neutrino bursts with the AMANDA detector, Astropart.Phys., 16, 345 (2002)

[17] B. Aharmim, S.N. Ahmed, A.E. Anthony et al., Low Multiplicity Burst Search at the Sudbury Neutrino Observatory, Astrophys.J., 728, 83 (2011)

[18] T. Lund, A. Marek, C. Lunardini et al., Fast time variations of supernova neutrino fluxes and their detectability, Phys. Rev. D 82, 063007 (2010)

[19] Bellini G. et al.(Borexino Collaboration), First real time detection of ${ }^{7}$ Be solar neutrinos by Borexino, Phys. Lett. B 658 (4), 101 (2007)

[20] Bellini G., Novel results on low energy neutrino physics, Talk at TAUP 2011 conference, Munich, 5-9 Sept. 2011,

[21] Eguchi K. et al. (KamLAND Collaboration), First Results from KamLAND: Evidence for Reactor Antineutrino Disappearance, Phys. Rev. Lett. 90, 021802 (2003)

[22] E.N.Alexeyev, V.V.Alexeyenko, Yu.M.Andreyev et al., Baksan underground scintillation telescope, in proceedings of 16 ICRC, Kyoto, 10, 276 (1979)

[23] Achkasov V.M., Bakatanov V.N., Novoseltsev Yu.F.et al., An investigation of the energy spectrum and inelastic muon interaction at the Baksan Underground scintillation telescope, Bull. Russ. Acad. Sci. Phys., 50, 2224 (1986)

[24] Imshennik V.S., Nadezhin D.K. Final stages of star evolution and supernova explosions, Itogi Nauki $i$ Tehniki, ser. Astronomy 21, 63 (1982)

[25] Hillebrandt W., Hoflish P., The supernova 1987A in the Large Magellanic Cloud, Rep.Prog.Phys. 52, 1421 (1989)

[26] Pantaleone J., Neutrino oscillations at high densities, Phys. Lett. B 287, 128 (1992)

[27] Sawyer R.F., Speed-up of neutrino transformations in a supernova environment, Phys. Rev. D 72 , 045003 (2005)

[28] Duan H., Fuller G.M., Carlson J. et al., Simulation of Coherent Non-Linear Neutrino Flavor Transformation in the Supernova Environment I: Correlated Neutrino Trajectories, Phys. Rev. D 74, 105014 (2006)

[29] I. Tamborra, G. Raffelt, F. Hanke et al., Neutrino emission characteristics and detection opportunities based on three-dimensional supernova simulations, Phys. Rev. D 90, 045032 (2014)

[30] Imshennik V.S. Explosion Mechanism in Supernovae Collapse, Space Sci.Rev. 74, 325 (1995)

[31] Bajkov V., Suslin V.M., Chechetkin V.M. et al., Radiation of a neutrino mechanism for type II supernovae, Russ. Astronom. journal, 84 (4), 308 (2007)

[32] Adams S.M., Kochanek C.S., Beacom J.F. et al., Observing the Next Galactic Supernova, Astrophys.J., 778, 164 (2013) 